\title{
Extra-Uterine Gestation occurring twice in the same patient, with a Note on the Treatment of Tubal Mole.
}

\author{
By Becкwith Whттеноuse, M.S. (Lond.), F.R.C.S., Birmingham.
}

THE occurrence of tubal gestation twice in the same patient, although not a phenomenon of extreme rarity, is still of sufficient interest to warrant the recording of a case before this Society.

The patient, a 2-para, aged 29, was admitted to the General Hospital, Birmingham, on September 28 1913, complaining of acute abdominal pain and uterine hæmorrhage of three weeks duration, preceded by eight weeks' amenorrhoea. The attack was of sudden onset with romiting and fainting, and exactly resembled an illness two years previously when the left ovary and tube were removed by Dr. Thos. Wilson for left tubal gestation. After leaving the Hospital on that occasion she was quite well and menstruated regularly and normally until about eleven weeks before her present attack. On admission, her temperature registered $100^{\circ} \mathrm{F}$., the pulse was 108 , and a very low tension. The patient was anæmic, and on abdominal examination a large mass was felt on percussion. Per vaginam the uterus was found displaced to the left side of the pelvis by a large tender swelling, in the right posterior quadrant of the pelvis. There was some slight hrmorrhage from the uterus on examination.

A diagnosis of right tubal abortion was made, and laparotomy advised.

The operation was performed the same evening, the right uterine appendages being removed. The patient made an uninterrupted recovery.

At the operation it was noticed that the left appendages were absent, the Fallopian tube and ovarian ligament being represented by a stump $\frac{1}{4}$ in. long at the corresponding uterine cornu. No adhesions were present on this side. The adhesions in the right side of the pelvis were only recent.

The specimen, consisting of the right Fallopian tube and ovary, was dissected immedjately under warm saline solution. It shows a tubal mole in process of abortion and exhibits well the points to which Dr. Thomas Wilson and the writer drew attention in a communication read before the combined meeting of Societies in May 1913. The points to which emphasis is directed are-(1) the oval shape of the mole with a constriction near the outer extremity produced by the margins of the ostium abdominale; (2) the longitudinal sulci upon the surface of the mole produced by the folds of the tubal mucosa; (3) the narrow attachment of the mole to the floor of the tube by a sessile base measuring only $\frac{1}{2}$ inch in diameter; (4) the position of the basis of attachment at the proximal end of the 
gestation, the hæmorrhage thus taking the path of least resistance and flowing in the direction of the ostium abdominale.

Remarks.

The case raises the important point as to whether we are justified in removing a uterine appendage in cases of tubal mole or incomplete abortion? Personally, I believe it is not good surgery to sacrifice the ovary and tube in these instances, and that it is rather of the nature of a useless mutilation, which may well be replaced by more conservative measures. Dissection under normal saline has shown that the mole is only attached by a narrow base, and that no great difficulty need be anticipated in controlling the hæmorrhage. The mole may easily be reached by longitudinal incision through the wall of the tube. After enucleation and control of the bleeding point, if any, the wall may be sutured by a continuous suture of fine silk. The dilated condition of the tube in these cases will render the introduction of the suture not particularly difficult. I have performed this procedure upon several occasions on specimens removed at operation, and I am convinced that it may prove of use in cases such as the present, where successive tubal pregnancies have meant the loss of both uterine appendages. Objections to the proposed operation are that (1) total or fatal occlusion of the tube may be produced which will favour the subsequent occurrence of further ectopic gestation, should transmigration of ovum or spermatozoon take place; (2) owing to the distension of the tube with absence of hypertrophy, it is possible that the tissues may not return to the normal condition, and that a structure is left which may not only be functionless, but also favour the production of hydrosalpinx and other inflammatory lesions.

As regards the first objection, I do not see that the risk of occlusion of the tube is as great in cases of tubal mole as in inflammatory conditions which are from time to time subjected to operation by salpingectomy. Furthermore, I do not know that tubal gestation is more common after these operations than in patients where no cause can be found. Concerning the second objection, it is remarkable how quickly and how perfectly tissues return to the healthy and normal condition, as witness the disappearance of widespread adhesions in some cases of pyosalpinx and pelvic abscess. Muscle is present in the wall of the pregnant tube, and although hypertrophy is absent, it is not unreasonable to suppose that complete involution may be effected by this means. That the muscle is functionally active is shown by the occasional observance of peristalsis in the wall of a pregnant tube the seat of partial abortion.

The objections therefore do not appear to be weighty, and certainly do not contraindicate an attempt at the performance of conservative salpingotomy when the occurrence of tubal mole or partial tubal abortion appears to call for the procedure. 